\title{
Article \\ Methodology to Improve Services in Small IT Centers: Application to Educational Centers
}

\author{
Juan Luis Rubio Sánchez
}

Citation: Rubio Sánchez, J.L.

Methodology to Improve Services in Small IT Centers: Application to Educational Centers. Computers 2021, 10, 8. https://doi.org/10.3390/ computers10010008

Received: 27 November 2020 Accepted: 30 December 2020 Published: 4 January 2021

Publisher's Note: MDPI stays neutral with regard to jurisdictional clai$\mathrm{ms}$ in published maps and institutional affiliations.

Copyright: () 2021 by the author. Licensee MDPI, Basel, Switzerland. This article is an open access article distributed under the terms and conditions of the Creative Commons Attribution (CC BY) license (https:// creativecommons.org/licenses/by/ $4.0 /)$.
Escuela de Ciencias e Ingeniería, Universidad a Distancia de Madrid, 28400 Collado Villalba, Spain; juanluis.rubio@udima.es

\begin{abstract}
Educational centers (schools, academies, high schools, etc.) are usually small companies, which make them special in terms of management. The management of IT services is far from standard and based in home solutions. The disadvantage of this approach is clear, as it happened during the COVID-19 pandemic period. The solution to properly managing IT services is based on the use of the ITIL (Information Technology Infrastructure Library). The question is how to apply this standard that only defines the processes to implement, but does not describe the way or the order to implement them. In this article it is shown which IT processes are really needed in any educational center and the order in which they should be implemented. The method used consists of fulfilling a knowledge database with extensive information from schools, academies, and other educational centers. After that, an existing optimization model is adopted and a representative learning center is defined, which is used to propose the IT processes sequence; finally, a set of optimal IT processes and the order to implement them is defined. These ordered processes optimize the quality of IT for learning services. The main result is an ordered set of IT processes that best fit the needs of IT departments in small educational centers.
\end{abstract}

Keywords: IT service; process; implementation sequence; educational center

\section{Introduction}

Since the appearance of computers, more and more companies have been introducing technology in their processes, in particular information and communication technologies. Both new and old companies need to relay their management and production in computers. But this is not an easy transformation for all companies. This transformation needs to be based on standards or best practices handbooks. Furthermore, the current COVID19 pandemic period is forcing all companies to move to online mode, even if they are not prepared for it or they do not have IT departments specifically adapted to online services. The implications of COVID-19 in terms of remote services and teleworking are affecting countries in different ways, as pointed in [1] where it indicates "in Sweden and the Netherlands more than $60 \%$ of workers in knowledge-intensive business services were teleworking, this fraction was below 30\% in Italy, and even lower in Austria and Germany." This article also clarified that there are great differences in the prevalence of remote services in education (for example e-learning) among Northern and Central-Southern European countries. The percentage in other Southern European countries (Spain, Portugal, Greece) is also below 30\%: the situation is aggravated in these countries because of the reduced size of the companies [2].

The services offered from IT departments in face-to-face learning centers are usually focused on back-office issues, but they are not affected by the massive demand of services. Typical standard services consider qualifications, payments, scheduling, etc., but online education demands much more services due to the massive consumption of information (online books, exercises, etc.) by students. This increase in services cannot be attended to if there is not a set of processes to cover all IT tasks. This situation becomes critical if 
educational centers are small or very small, which happens frequently in countries such as Spain (and other Southern European countries) where more than $95 \%$ of companies are small or very small (less than 10 employees) [3].

It is a fact that the most used framework for the management of IT services is Information Technology Infrastructure Library (ITIL) [4]. But as ITIL is the most used, there exists a lack of definitive information about how to implement the processes defined in it and which order of processes should be followed (the complete list of ITIL processes can be found in Appendix A Table A1). This represents a handicap mainly for small organizations with few resources and small data centers or small IT departments. Different authors proposed examples of approaches to the problem of strategy, sequence of processes, and success factors when implementing ITIL processes [5-9]. These references evidence that ITIL implementation is usually analyzed from diverse points of view.

It is also common to analyze the application of ITIL in specific regions $[8,10]$, in specific industries [11,12], in a specific sector (public/private) [13], or in a specific type of company [14].

Based on the evidence, which shows that it is a common practice to analyze the implementation of ITIL on specific types of organizations (private, public, big companies, small companies, located in a concrete region, or any other criteria), and considering that ITIL implementation is analyzed from diverse points of view (order of processes, methodology, success factors, etc.), this article focuses on the educational industry in Spain and the set of ordered processes needed to optimize the implementation of ITIL. This ordered set of ITIL processes enhances the quality of IT services (once implemented) and eases the digital transformation obliged by COVID-19.

First, an exhausted systematic review was developed to understand the alternatives for a proper implementation of processes in IT departments; with this information about the state of the art, a proposal for implementing ITIL in small educational centers is shown. Finally, a comparative of this sequence with other solutions is shown.

\subsection{Approach 1: Fixed Set of Processes}

Fixed sequences of IT processes refer to strategies that propose a static sequence of ITIL processes independent of the company and the competitors, such as the processes already implemented or any variables. Examples can be found in [9] and in [15]. The proposed solutions look for a measure of the relationship among different processes. The solutions proposed in $[9,15]$ identify the dependencies between every process and the rest of the processes. The number of dependencies of a particular process is an indicator of its importance. This methodology allows for the identification of a set of critical processes, which should be implemented at the start. This set of processes is a fixed sequence, as it only depends on the processes (not on the company). The disadvantage of the proposal is that it is not extended to all ITIL processes, but rather only to a few of them.

The notion of a fixed sequence comes up again in [16]. In this article, ITIL implementation is presented from the definition of three models:

- A first model of isolated ITIL processes;

- Another model that contains the dependencies between ITIL processes; and

- A final model to relate the capacity level of the processes and the maturity level of the organization. One of the main characteristics of this proposal is the combination of concepts of ITIL processes and CMMI (Capability Maturity Model Integration) to propose a sequence. For example, the sequence of ITIL processes is built with the help of CMMI to know the maturity of each ITIL process in the organization.

A static order of implementation is also presented in [17]: There is no explicit justification but it shows a sequence that could be used as a reference. That is, all interested schools or centers can use an identical sequence to the one presented in the proposal because company characteristics are not taken into account. It does not consider the size of the IT department or the size of the company: The proposed sequence is the same. 
In other proposals, $[18,19]$, the aim is just to highlight a starting point: The goal is to define the first ITIL process to begin the implementation. The authors offer empirical basis supported by deep market research. The number one process to be implemented is IM (incident management), and after that, SLM (service level management) and SCM (service catalog management): This proposal can be applied to any small IT center (including schools and training centers). These approaches are referred to as strategies that propose a very short static sequence (usually just one process, and sometimes up to three processes) that do not depend on the company, on the competitors, on the processes already implemented, or on any parameters.

The author of [20] offers a static sequence of processes (it does not generate the complete list of ITIL processes, but rather only a few) to implement in small companies considering the implementations documented by other authors and validating the set of criteria to sequence processes with ITIL experts. It is relevant [20] because it is specifically oriented to small and medium companies.

The approach exposed in [21] determines a fixed sequence of ITIL processes that takes into account elements as the tasks needed to implement every process, the distribution of information, and the flow of data between every ITIL process and the dependencies among all processes. This reference is interesting because it offers a complete sequence of processes (to be implemented) instead of just a short set of processes without prioritization.

An approach to generate quick benefits during ITIL implementation can be found in [22], where a fixed set of processes is proposed. This proposal offers a set of ITIL processes to start with, independent of any consideration of the company or any consideration of the IT department.

\subsection{Approach 2: Non-Fixed Set of Processes}

Several authors have worked on determining a dynamic (i.e., non-fixed) ordered set of processes. An example in this area is [5]. It explains a model to sequence ITIL processes for implementation in any organization, based on fuzzy logic. The model is built taking into consideration that the sequence of processes depends on technical and organizational factors: These are subjective factors. The approach is oriented to select the criteria that are be used to define the sequence of ITIL processes. The criteria are determined by the opinion of a set of ITIL experts. These opinions are treated with fuzzy techniques to translate the ambiguity of opinions into numerical values. The methodology has an significant disadvantage: It does not consider important characteristics of the company that is implementing ITIL processes, such as size, number of employees, geographical area, etc. A similar proposal also based on the use of fuzzy logic is presented in [23].

Another work with a non-fixed sequence was published by [7]. The author explains a method where the processes that provides "early wins" are the first ones to be selected. The problem in this method is to clarify what "early wins" are. The solution to defining "early wins" is found through customer satisfaction surveys. The author also explains how to define the processes that should be implemented in the long term (from the results of the surveys). This method is suitable for any company, but it has the disadvantage of the sequence being driven by client's opinion: It is necessary to request the responses from the clients and the processes are oriented to satisfy only the needs of the clients (there are other users of IT services, for example, the workers).

The approach explained in [6] is based on the Technology Adoption Model (TAM). This model describes how to adopt ITIL in any organization: The sequence of ITIL processes is dependent on requisites of the organization but prioritizes "critical" processes. A similar approach can be found in [8], where the authors expose a study of the reasons for implementing ITIL and the processes that should be initially implemented: The key factors for implementation are the size of the company, the industry, and the geographical area.

Finally, an approach for a dynamic sequence is [24]. This proposal offers an ITIL sequence of processes that takes into consideration the characteristics of the company, the competitors, and the objectives of the company. On the one hand, the model considers 
the main parameters of the company that is implementing ITIL processes; on the other hand, it involves in the model the level of implementation of the competitors, and finally, it returns the sequence that best fits the aims of the company in the implementation of ITIL. This makes this methodology be considered dynamic because it builds a specific sequence for every company.

\subsection{Approach 3: Success Factors and Other Approaches}

The implementation of ITIL is considered in different ways. There is a group of authors who tried to identify "success factors" in the implementation of ITIL. An example is [25], where a survey deployed in different enterprises was considered to understand the factors that may affect the success of ITIL implementation. Another example is found in [26]: In this case, specific success factors were proposed and analyzed-how the involvement of management affects the implementation of ITIL, how the organization's commitment may help the implementation of ITIL processes, and how effectiveness can help the implementation of ITIL. The authors in [26] showed a set of items that allowed for the success of an ITIL implementation, which were similar to the success factors addressed by the authors in [25]. However, in this case, the conclusions were obtained from a set of 160 companies. It is accepted that the study was limited to a specific geographical area and cannot be considered valid for any geographical area.

A set of articles has also been identified that provides methods and tactics to implement ITIL processes. An example is [27]: In this reference, the author analyzes which steps should be considered when implementing ITIL (considering four big organizations). Taking this experience as a basis, success factors are identified to reach a successful implementation of ITIL processes. It also indicates the starting process-the incident management process-but it does not indicate any sequence from this first process.

An alternative to these key factors is exposed in [28]. In this case the most important elements to success are the involvement of management and the use of software tools. It explains the great importance of the implementation task: Success depends on the use of the correct software tools that ease the management of the implementation. This topic is also covered in [29], where business process management (BPM) tools are proposed for managing ITIL implementation.

A vast systematic review about success factors is presented in [30]. In this case, the proposal to identify the critical success factors uses the AHP (analytical hierarchical process) methodology. This methodology allows one to evaluate, calculate the average, and find inconsistencies in a set of opinions given by experts.

Business process change (BPC) is a methodology explained in [31]. This methodology determines the set of success factors (it does not clarify anything about its order of implementation). It is possible to find other methodological approaches to implementing ITIL in [32-34]: These references propose methods to help the implementation of ITIL by selecting the first process to implement.

A completely different approach is shown in [35], where a set of failure factors is presented. These elements make the implementation of ITIL difficult. Something similar occurs in [36], where the authors analyze the challenges to implementing ITIL. A similar argument is found in [37], where the challenges of an ITIL implementation are explained.

\subsection{A Specific Approach for Small Data Centers in Educational Companies}

Once the main alternatives are reviewed, it can be deduced that the approach that takes into consideration the characteristics of the center and the characteristics of the market and competitors is [24]. This proposal builds a sequence of processes based on a mathematical model that uses market information to decide whether the school or center is in a better or worse position than the competitors. To decide the sequence, a "relative position" factor is defined and used to order the processes, which ends with an optimal sequence. In addition, it offers an online tool to build the best sequence to help schools and training centers obtain the sequence. 
The implementation of these processes will hopefully help centers to offer better IT services to students, teachers, and the rest of the staff. The next section explains the data acquisition and the application of the methodology in a standard small education center.

\section{Materials and Methods}

In order to obtain knowledge about the current implementation level of ITIL in training and education companies, a survey (web-based) was designed; data was stored in the public site included in the Supplementary Materials section. The survey was distributed by email and, when needed, completed by face-to-face interviews. The answers were reviewed to avoid including more than one answer from the same center.

Once the data was gathered in the database it was possible to apply the methodology [24] to any company. This could be done by defining the characteristics of the company and introducing these characteristics to the model (also available on the cited website). In particular, the methodology was applied to a generic small educational center that was representative of any small educational center. This can hopefully help small educational centers (with small IT departments) improve their services.

The next diagram (Figure 1) represents the methodology applied [24]: (1) The data gathered were stored in a database. These data were mainly the characteristics of the centers and their levels of implementation of every ITIL process. (2) Data from a generic center was required as well as the parameters used to generate the optimal set and sequence of processes. (3) The mathematical model was applied to obtain the set of ITIL processes (for their implementation) and their order (in which they should be implemented).

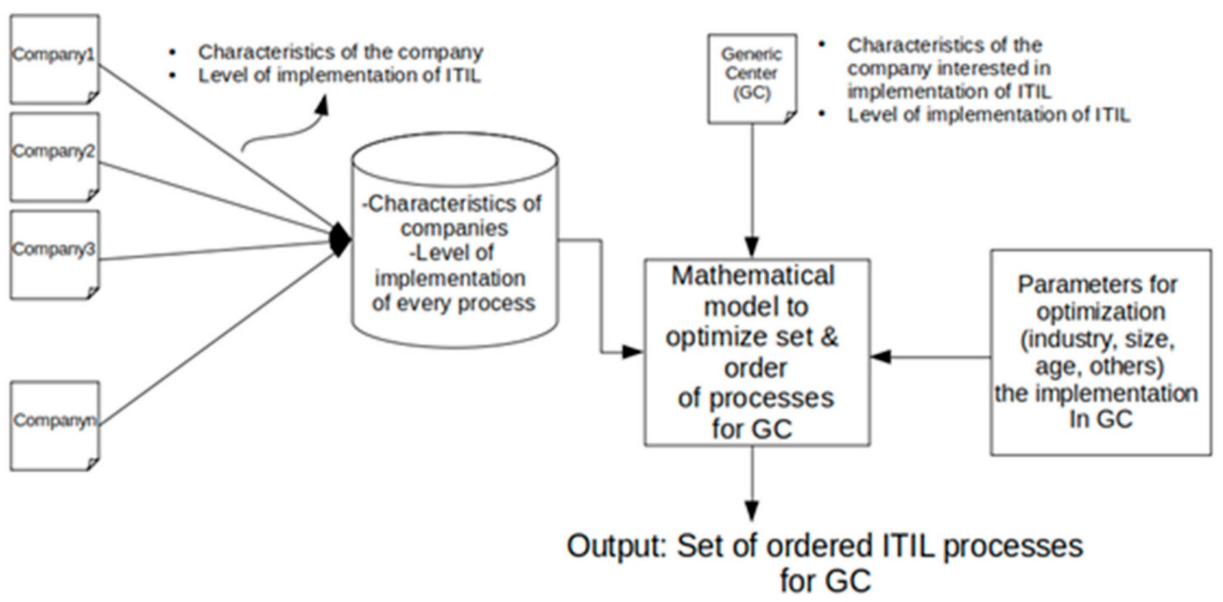

Figure 1. Schema of materials and methods applied.

\subsection{Scope}

The scope of the study was any company in the educational industry operating in Spain considered a small company (the limit to be considered a small company in Spain is 50 employees). The poll was used to collect data about the level of implementation of ITIL processes in the mentioned companies as well as about the characteristics of the companies (age, size, etc.).

The companies were randomly selected. A subset of 200 companies was sent a message to participate in the survey.

The poll was sent to the managing director or the IT director (if possible) and responses were accepted from 20 September until 20 October.

\subsection{Database}

The database stored information about characteristics of the school or training center (size, number of employees, number of IT employees, area where the company operates, industry, and other). After these questions, the centers were asked about the level of 
implementation of every process in the ITIL standard; that is, it was desired to know whether the IT department had implemented every process presented in the ITIL standard. The possible answers were:

1. The process is not implemented and it is not planned (or it is an unknown process for the company).

2. The process is not implemented but it is planned in the medium term.

3. The process is implemented or it will be implemented in the short term.

Once the database was filled, the data stored in it could be used in conjunction with the model [24] to generate an ordered set of processes.

\subsection{Technical Issues}

The method to select companies was by a random one-phase process. Once the questionnaire was closed, the number of responses was 101. The results from the survey offered a confidence level of over $90 \%$ and a sampling error of $8.5 \%$. The number of responses guaranteed that no overlapping was produced in the answers.

The next table (Table 1) summarizes the parameters of the survey.

Table 1. Description of the survey.

\begin{tabular}{cc}
\hline Concept & Value \\
\hline Scope & Spain \\
\hline Universe & $\begin{array}{c}\text { Educational centers with less than } \\
10 \text { full-time employees }\end{array}$ \\
\hline Type of polling & Web form \\
\hline Sent & 200 \\
\hline Received & 101 \\
\hline Minimum to be significant & 64 \\
\hline Confidence level & $90 \%$ \\
\hline Error & $<8.5 \%$ \\
\hline Company selection & Random \\
\hline Mode & One phase
\end{tabular}

\section{Results}

To illustrate what was previously explained, the procedure cited in [24] was applied. The methodology was applied to a highly representative case. This representative case was a typical small school or small training center with a set of teachers, staff, and a small IT department composed of five people [3]. This small center was located in Spain and was characterized by a low degree of implementation of ITIL processes. It could be assumed that capacity management, availability management (partially), and access management, continuous improvement (partially) were the only processes implemented, as reported in the survey by the small training centers. This center was representative of most small educational centers (schools, small universities, high schools, academies, etc.). This standard small center was used as input data in the methodology exposed in [24]. This methodology requires a criterion to be defined to get an optimized sequence of processes for the IT department: either optimization by size of the company, by IT size, by age of the center, by geographical area, or by industry (in this case the industry is education), or a general optimization (that considers all possible criteria). An interest in improving technology services was assumed, taking as a reference centers in the educational industry, but with no special consideration for age, region, or any other characteristic (that is, a general optimization case).

This is quite important because, as described in [24], the optimization could be oriented to obtain a sequence of processes to get closer to competitors, to get closer to generic small 
companies, etc., in terms of ITIL implementation. In this case, as cited before, no special criteria were followed to optimize.

In this case, the application of the methodology indicated produced the next optimal order of processes to be implemented (only the 11 first processes are shown):

1. Service catalog management

2. Incidence management

3. Financial management

4. Requirement fulfillment

5. Service portfolio management

6. Transition management

7. Testing management

8. Release and deploy management

9. Asset management

10. Supply management

11. Change management

This result indicates that an IT department needs initially to establish the list of services offered by IT. That is, the best way to improve quality in IT is to publish the services that can be developed. This makes sense because it avoids many incidences from users who do not know what can be required and what cannot be required of the IT department. In second place, the management of incidences addresses the need for gathering all incidences and treating them in a unified procedure. This makes users satisfied because their demands are attended to. In third place, financial management clarifies which projects can be attended to and which ones cannot. The correct scheduling and cost estimation for every task is a common problem in IT departments: This process establishes a common rule to evaluate IT projects so IT departments know in advance the resources they need to develop all projects. The rest of the processes considered operative tasks as testing, release, and deployment, or change management.

In order to analyze whether the sequence of implementation was similar to other ones proposed by the authors previously mentioned, a double test was used: Spearman and Kendall contrasts.

It is important to state that the objective was not to compare the benefits of the sequences: The procedure explained in [24] to get the optimal sequence was based on the distance between the implementation degree of ITIL in a particular company and the implementation degree of ITIL in a group of companies (similar in size, age, industry, IT department size, etc. The procedure lets you choose the characteristics that should be considered to get the sequence). Considering the characteristics of each alternative explained in 1.1, 1.2, and 1.3, the sequence from [21] was the one that offered a complete set of ordered ITIL processes. The rest of the alternatives did not offer a complete ordered sequence: They only offered a partial subset of ITIL processes. Both sequences (calculated and the reference to compare) are included in Table 2.

Table 2. Sequence calculated and reference sequence.

\begin{tabular}{ccc}
\hline Order & Sequence Calculated [24] & Reference Sequence [21] \\
\hline 1 & Service catalog management & Change management \\
\hline 2 & Incidence management & $\begin{array}{c}\text { Release and deploy } \\
\text { management }\end{array}$ \\
\hline 3 & Financial management & Asset management \\
\hline 4 & Requirement fulfillment & Incidence management \\
\hline 5 & Service portfolio management & Service level management \\
\hline 6 & Transition management & Security management \\
\hline 7 & Testing management & Testing management
\end{tabular}


Table 2. Cont.

\begin{tabular}{ccc}
\hline Order & Sequence Calculated [24] & Reference Sequence [21] \\
\hline 8 & $\begin{array}{c}\text { Release and deploy } \\
\text { management }\end{array}$ & Event management \\
\hline 9 & Asset management & Service catalog management \\
\hline 10 & Supply management & Availability management \\
\hline 11 & Change management & Financial management \\
\hline
\end{tabular}

It was necessary to verify that the calculated optimized sequence and the reference sequence were not correlated; that means they were independent, otherwise the optimized one could not be accepted as a really new sequence. Therefore, considering the sequence proposal in [21] as a reference, the following definitions are proposed:

Ho: null hypothesis: both sequences are independent (i.e., both order of processes are significantly different)

H1: alternative hypothesis: both sequences are dependent (i.e., it is not possible to affirm that both order of processes are independent)

Table 3 illustrates the results for both tests with different confidence levels:

Table 3. Test for the independence of the proposed sequence.

\begin{tabular}{|c|c|c|c|}
\hline & $\begin{array}{c}\text { Confidence Level } \\
\alpha=0.05\end{array}$ & $\begin{array}{c}\text { Confidence Level } \\
\alpha=0.10\end{array}$ & $\begin{array}{c}\text { Confidence Level } \\
\alpha=0.20\end{array}$ \\
\hline Spearman contrast & $\begin{array}{c}\rho=0.285 \text { in } \\
(-0.425-0.425)\end{array}$ & $\begin{array}{c}\rho=0.285 \text { in } \\
(-0.361-0.361)\end{array}$ & $\begin{array}{l}\rho=0.285 \text { not in } \\
(-0.284-0.284)\end{array}$ \\
\hline Kendall contrast & $\begin{array}{c}\tau=0.143 \text { in } \\
(-0.307-0.307)\end{array}$ & $\begin{array}{c}\tau=0.143 \text { in } \\
(-0.264-0.264)\end{array}$ & $\begin{array}{c}\tau=0.143 \text { in } \\
(-0.203-0.203)\end{array}$ \\
\hline
\end{tabular}

It was observed that both contrasts resulted in accepting the null hypothesis for every alpha value (except the Spearman contrast for $\alpha=0.20$, where a limit value was obtained). This result led us to accept the independence of both sequences. As a consequence, the optimal sequence designed to get as close as possible to the competitors offered a different result than [21]. A deeper analysis determined the origin of these discrepancies, which are explained in next points:

- Validation and testing process, requirement fulfillment process, incidence management process, and financial management process were proposed to be implemented in the same position in the sequence (or quite close, not statistically different)

- The proposed sequence placed service catalog management as the first process to be implemented, whereas the sequence in [21] moved it to the ninth place.

- Change management was proposed in first place by [21], but it was proposed in 11th place by the optimal sequence.

- The proposed sequence placed service catalog management as the first process to be implemented, whereas the sequence used as reference moved it to ninth place.

- $\quad$ Service level agreement management, security management, and event management were proposed in the fifth, sixth, and 8th positions, respectively, by the sequence in [21], but they were placed further than 10th position by the optimal sequence.

- The most discrepancies appeared in availability management process and portfolio management process, which were placed in the 10th and 19th positions, respectively, by [21], but were proposed in the fifth and 22nd positions, respectively, by the optimal sequence. These two processes were the ones that most affected the value of the statistics $\rho$ and $\tau$. 


\section{Discussion}

Educational companies are usually small companies with very few employees dedicated to IT. This makes it really difficult to offer high-quality IT services. This is important because nowadays more and more centers need to offer online educational services (schools, adult schools, academies, etc.). One of the reasons is that small centers have only a few resources in the IT department and it is rather complicated to implement processes such as the ones defined in ITIL, which is, as mentioned before, the most extended and used standard in the management of IT departments. The consequence is that the quality of services is far from the desired level. This situation may decrease student satisfaction and, in the long term, may affect the survival of the company.

There are different approaches that help to implement ITIL in companies. These approaches can be categorized in three big groups: (a) a first group that identifies the key and failure factors in the project of implementing ITIL, (b) a second group that identifies a fixed set of processes and the first ITIL process to implement, and (c) a third group that identifies methodologies and finds sequences of processes specifically designed for each company.

The methodology exposed in [24] offers the possibility to adapt the result, that is, the ordered set of ITIL processes to be implemented, for every company. This possibility was used to apply the methodology in [24] to a general case of a small educational center.

The sequence obtained (see Table 2) considered that catalog management should be followed by operational processes such incidence management. This sequence solved most IT defects in terms of quality. The sequence was completed with up to 11 processes taken from the ITIL standard (representing more than $20 \%$ of the complete list of ITIL processes). The reason to consider this set of processes is that following Pareto's rule, the $80 \%$ of deficiencies in quality are caused by $20 \%$ of processes.

The sequence obtained was compared with [21] (it considered the complete list of ITIL processes and was validated by an expert panel). The results indicated an independence of the optimal sequence obtained by applying [24] and the sequence in [21]: This indicated that the optimal sequence proposed was different from the one taken as reference.

The advantage of this solution lays in the fact that it was specifically built for small educational centers instead of the proposals analyzed, which make sense in big organizations, or they represent general purpose sequences (i.e., not specific for educational centers). It is also possible to identify other benefits: The proposed set of processes was obtained by application of [24]. This methodology does not require the involvement of experts or consultants and so it is cheaper. Moreover, the set of ordered processes is built considering the data obtained from the competitors, optimizing the close-up to them.

On the other hand, the limitation of the methodology applied is the implementation itself. That is, there is no clue about how to implement every single process or what elements should be considered to succeed. The approach establishes the set and the order of the processes, but it does not explain how to implement a particular process. It is possible to take some ideas about it from the literature (mainly in the approaches cited in Section 1.3).

A second limitation of the present study is that it was developed with Spanish companies and the conclusions are valid in Spain (and possibly in other Southern European countries, but it should be verified).

This research can be exploited in the future in different ways. The first idea is to apply the methodology to other small companies related to the educational industry, for example, small companies that develop contents for e-learning, small companies that develop software for e-learning, or small web portals for the education community. A second way to continue with the research is to compare the results of ITIL implementation to centers that decided to use other standards to manage IT departments. That is, should a small center use ITIL, or would it be better to apply other standards? Finally, a third line to investigate is related to providers: When talking about such small centers, would it not be a better idea to externalize all IT activity and focus on the activity of the company just in education? It should be considered that any IT department implies the hiring of IT 
resources, servers, and software, and implementing processes, among other implications. Nowadays, it is possible to find solutions to externalize any service, which probably offer better services than internal IT departments.

Supplementary Materials: The following are available online at http://schoolprocesses.sytes.net or https:/ / sqitil.sytes.net database and online process sequencing tool.

Funding: This research received no external funding.

Institutional Review Board Statement: Not applicable.

Informed Consent Statement: Not applicable.

Data Availability Statement: Not applicable.

Conflicts of Interest: The authors declare no conflict of interest.

\section{Appendix A}

Table A1 includes the list of ITIL processes used in the poll.

Table A1. List of ITIL processes.

\begin{tabular}{cccc}
\hline Number & Value & Number & Value \\
\hline 1 & $\begin{array}{c}\text { Strategic management of } \\
\text { services }\end{array}$ & 14 & Transition schedule \\
\hline 2 & Portfolio management & 15 & Change management \\
\hline 3 & Financial management & 16 & $\begin{array}{c}\text { Deploy and version } \\
\text { management }\end{array}$ \\
\hline 4 & Demand management & 17 & $\begin{array}{c}\text { Validation and test } \\
\text { management }\end{array}$ \\
\hline 5 & Business relation management & 18 & Asset and configuration \\
management & Change management \\
\hline 6 & Design coordination & 19 & Knowledge management \\
\hline 7 & Service catalog management & 20 & Problem management \\
\hline 8 & Availability management & 21 & Incident management \\
\hline 10 & Service level management & 22 & Access management \\
\hline 11 & Continuity management & 23 & Event management \\
\hline 12 & Security management & 24 & Request management \\
\hline 13 & Provider management & 25 & Continuous improvement \\
\hline & & 26 &
\end{tabular}

\section{References}

1. Milasi, S.; González-Vázquez, I.; Fernández-Macías, E. Telework in the EU before and after the COVID-19: Where We Were, Where We Head to; Science for Policy Briefs; European Commission: 2020. Available online: https://ec.europa.eu/jrc/sites/ jrcsh/files/jrc120945_policy_brief_-_covid_and_telework_final.pdf (accessed on 3 January 2021).

2. Moral, E. Growing by learning: Firm level evidence ont size-productivity nexus. In Documentos de Tabajo-N ${ }^{\circ} 1613$; Banco de España: Madrid, Spain, 2016; ISSN 1579-8666. [CrossRef]

3. Urueña, A.; Ballestero, M.P.; Castro, R.; Cadenas, S.; Maira, M.; Prieto, E. Análisis Sectorial de la Implantación de las TIC en las Empresas Españolas; Informe ePYME; Ministerio de Asuntos Económicos y Transformación Digital: Madrid, Spain, 2019; ISSN 2341-4030.

4. Gervalla, M.; Preniqi, N.; Kopacek, P. IT infrastructure library (ITIL) framework approach to IT governance. IFAC PapersOnLine 2018, 51, 181-185. [CrossRef]

5. Zare, A.; Mansouri, T.; Mehrabioun, M. Supporting the module sequencing decision in ITIL solution implementation: An application of the fuzzy TOPSIS approach. Int. J. Inf. Technol. Syst. Appoach 2014, 7, 41-60. [CrossRef]

6. Mehravani, S.; Haghighinasan, M.; Hajjiheydari, N. ITIL Adoption Model Based on TAM. International Conference on Social Science and Humanity 2011; IACSIT Press: Singapore, 2011. 
7. Menken, I. ITIL V3 Implementation Quick Guide—The Art of Stress-Free IT Service Management; Emereo Pty Ltd.: London, UK, 2009; ISBN 978-1-921573-41-5.

8. Marrone, M.; Gacenga, F.; Cater-Steel, A.; Kolbe, L. IT Service Management: A Cross-national Study of ITIL Adoption. Commun. Assoc. Inf. Syst. 2014, 34, 49. [CrossRef]

9. Arcilla, M.; Calvo-Manzano, J.; Cuevas, G.; Gómez, G.; Ruiz, E.; San Feliú, T. A Solution for Establishing the Information Technology Service Management Processes Implementation Sequence. In Software Process Improvement. EuroSPI 2008. Communications Computer Information Science; O'Connor, R.V., Baddoo, N., Smolander, K., Messnarz, R., Eds.; Springer: Berlin/Heidelberg, Germany, 2008; Volume 16.

10. Polard, C.; Cater-Steel, A. Justifications, Strategies, and Critical Success Factors in Successful ITIL Implementations in U.S. and Australian Companies: An Exploratory Study. Inf. Syst. Manag. 2009, 26, 164-175. [CrossRef]

11. Tan, W.-G.; Cater-Steel, A.; Toleman, M.; Seaniger, R. Implementing centralized IT service management: Drawing lessons from the public sector. IFAC PapersOnLine 2007, 51, 181-185.

12. Tawar, T. Applying ITIL Based Software Inside University's ICT Centre toward Continual Service Improvement. In Proceedings of the Information Systems International Conference (ISICO), Bali, Indonesia, 2-4 December 2013; Volume 2.

13. Orlandi, T.; Santos Dantas, F.; Souza Neto, J. The use of portfolio management for strategic alignment-A survey with Brazilian Companies. J. Inf. Syst. Technol. Manag. 2020, 17. [CrossRef]

14. Schaefer, J.; Baierle, I.; Nara, E.; Benitez, G.; Haetinger, C. Corporate Governance and Information Technology: A Hybrid Vision Based on COBIT and ITIL for Small Companies. In Proceedings of the XL Encontro Nacional de Engenharia de producao, Foz do Iguaçu, Brazil, 20-23 October 2020.

15. Arcilla, M.; Ruiz, E.; Cerrada, C.; Gómez, G.; Calvo-Manzano, J.A.; San Feliu, T.; Sánchez, A. Una Propuesta Organizativa de Los Procesos de SD y SS en ITIL. REICIS 2007, 3, 6-20.

16. Pastuszak, J.; Czarnecki, A.; Orlowski, C. Ontologically Aided Rule Model for the Implementation of ITIL Processes. In Proceedings of the 16th International Conference on Knowledge-Based and Intelligent Information and Engineering Systems, San Sebastian, Spain, 11-12 September 2012.

17. Lucio-Nieto, T.; Colomo-Palacios, R.; Soto-Acosta, P. Implementing an IT service information management framework: The case of COTEMAR. Int. J. Inf. Manag. 2012, 32, 589-594. [CrossRef]

18. Ayat, M.; Sharifi, M.; Sahibudin, S.; Ibraim, S. Adoption Factors and Implementation Steps of ITSM in the Target Organizations. In Proceedings of the Third Asia International Conference on Modeling \& Simulation, Bali, Indonesia, 25-29 May 2009. [CrossRef]

19. Calvo, J.A.; Lema, L.; Arcilla, M.; Rubio, J.L. How small and medium enterprises can begin their implementation of ITIL? Rev. Fac. Ing. Univ. Antioq. 2015, 77, 127-136. [CrossRef]

20. Lema, M.L. ITIL in Small to Medium-Sized Enterprises: Toward a Proposal Based on an ITIL Processes Implementation Sequence and a Profile Scheme Strategy for Implementing the First Process in the Sequence. Master's Thesis, Polytechnic University of Madrid, Madrid, Spain, 2015.

21. Miller, A. Implementation Priority of the ITIL Framework Based on a Process Sequencing Model; Doctoral Praxis-The George Washington University (ProQuest: 10251867); ProQuest LLC: Washington, DC, USA, 2017.

22. Case, G. ITIL V3: Where to Start \& How to Achieve Quick Wins. Available online: http:/ /www3.pinkelephant.com/articles / itilv3wheretostarthowtoachievequickwins.pdf (accessed on 3 January 2021).

23. Zarrazvand, H.; Shojafar, M. The Use of Fuzzy Cognitive Maps in Analyzing and Implementation of ITIL Processes. arXiv 2012, arXiv:1206.2297.

24. Rubio, J.L.; Arcilla, M. How to Optimize the Implementation of ITIL through a Process Ordering Algorithm. Appl. Sci. 2019, 34. [CrossRef]

25. Cater-Steel, A.; Tan, W.G. Implementation of IT Infrastructure Library (ITIL) in Australia: Progress and success factors. In Proceedings of the IT Governance International Conference, Auckland, New Zealand, 11-13 May 2005.

26. Iden, J.; Eikebrokk, T. The impact of senior management involvement, organizational commitment and group efficacy on ITIL implementation benefits. Inf. Syst. E-Bus. Manag. 2015, 13, 527-552. [CrossRef]

27. Koshravi, H.; Wang, J. How to Implement ITIL Successfully? In What Steps are Necessary to Implement ITIL and What Factors are Critical within Implementation Process; LAP Lambert Acad. Publications: Koln, Germany, 2011.

28. Eikebrokk, T.; Iden, J. ITIL Implementation: The Role of ITIL Software and Project Quality. In Proceedings of the $201223 \mathrm{rd}$ International Workshop on Database and Expert Systems Applications (DEXA'12), Vienna, Austria, 3-7 September 2012; IEEE Computer Society: Washington, DC, USA, 2012; pp. 60-64. [CrossRef]

29. Mahy, Y.; Ouzzif, M.; Bouragba, K. Supporting ITIL processes implementation using business process management systems. In Proceedings of the Third International Conference on Systems of Collaboration (SysCo), Casablanca, Morocco, 28-29 November 2016; pp. 1-4. [CrossRef]

30. Ahmad, N.; Noha, T.A.; Qutaifan, F.; Alhilali, A. Technology adoption model and a road map to successful implementation of ITIL. J. Enterp. Inf. Manag. 2013, 26, 553-576. [CrossRef]

31. Pedersen, K.; Kræmmergaard, P.; Lynge, B.C.; Schou, C.D. ITIL implementation: Critical success factors a comparative case study using the BPC framework. J. Inf. Technol. Case Appl. Res. 2010, 12, 11-35. [CrossRef]

32. Fry, M.M. ITIL Lite: A Road Map to Full or Partial ITIL Implementation; The Stationery Office: Norwich, UK, 2010. 
33. Binders, Z.; Romanovs, A. ITIL self assessment approach for small and medium agencies. Inf. Technol. Manag. Sci. 2014. [CrossRef]

34. Bon, J.; De Jong, A.; Kolthof, A.; Pieper, M.; Tjassing, R.; Van der Veen, A.; Verheijen, T. Gestión de Servicios TI Basada en ITIL; Van Haren: Hertogenbosch, the Netherland, 2016.

35. Shang, S.; Lin, S. Barriers to implementing ITIL-a multi-case study on the service-based industry. Contemp. Manag. Res. 2010, 6 , 53-72. [CrossRef]

36. Sebaaoui, S.; Lamrini, M.; Bouayad, A.; El Abbadi, L. Challenges of ITIL Implementation Approaches. In Proceedings of the 4th International Conference on Business Intelligence (CBI'18), Beni Mellal, Morocco, 25-27 April 2018.

37. Müller, S.; De Lichtenberg, C. The culture of ITIL: Values and implementation challenges. Inf. Syst. Manag. 2018, 49-61. [CrossRef] 